\title{
The antithrombotic effects of onion filtrates in rats and mice
}

\author{
Kanae Hyodo ${ }^{1}$, Izumi Horii ${ }^{1}$, Masaru Nishino ${ }^{2}$, John C Giddings ${ }^{3}$, Junichiro Yamamoto ${ }^{1 *}$ \\ ${ }^{1}$ Laboratory of Physiology, Faculty of Nutrition, Kobe Gakuin University and Cooperative Research Center of Life Sciences, Kobe \\ Gakuin University, Kobe, Japan; *Corresponding Author: yamamoto@nutr.kobegakuin.ac.jp \\ ${ }^{2}$ Hyogo Prefectural Technology Center for Agriculture, Forestry and Fisheries, Awaji Agricultural Technology Institute, Awaji, Japan; \\ ${ }^{3}$ Wales College of Medicine, Cardiff University, Cardiff, UK.
}

Received 10 February 2011; revised 29 March 2011; accepted 1 April 2011.

\begin{abstract}
The prevention of arterial thrombotic disease has a high priority in developed countries. An inappropriate diet is known to enhance the risk for acute thrombotic events, and a regular diet with proven antithrombotic effects might be a beneficial way to prevent disease. The present study was undertaken as part of a series of investigations to examine various vegetables and fruits for antithrombotic activity, and to add to the previously reported data on different onion varieties produced in the northern part of Japan (Hokkaido). For this purpose, a sophisticated method to assess shear-induced platelet reactivity/thrombolysis in vitro (The Global Thrombosis Test, GTT) was used to screen 5 different varieties of onion produced in the middle part of Japan (Awaji Island). The different onion varieties demonstrated a variable effect on thrombosis, and one particular variety, designated ONA-03, appeared to exert an antithrombotic effect. Another variety, ONA-01, appeared to have prothrombotic activity by inhibiting spontaneous thrombolytic activity. The especially effective variety was further investigated using an in vivo, laser-induced thrombosis model in mice. The heat stable antithrombotic activity in vitro and in vivo was demonstrated to be due to antiplatelet activity. The present findings added to the list of antithrombotic fruits and vegetables.
\end{abstract}

Keywords: Cardiovascular Disease; Stroke; Atherothrombosis; Onion; Quercetin

\section{INTRODUCTION}

The prevention of "lifestyle-related atherothrombotic diseases" such as myocardial infarction and stroke has become an important and urgent social task in many developed countries. Studies have provided clear evidence that an inappropriate diet, such as the Westernstyle high fat diet, plays a causative role in the pathogenesis and clinical outcome of thrombotic diseases [1].

The so-called French Paradox and red wine hypothesis $[2,3]$ has prompted many laboratory studies on antithrombotic fruits and vegetables, and epidemiological studies have provided evidence that intake of fresh fruits and vegetables could help to prevent cardiovascular disease and stroke [4-7].

Platelets play a pivotal role in arterial thrombotic diseases. Platelet-function in vitro is commonly assessed using platelet aggregometry, which measures platelet aggregation induced by various chemical agonists. In clinical practice, however, beneficial tests for the diagnosis and treatment of patients with thromboembolic disorders remain to be fully defined. Tests using native, non-anticoagulated blood in the presence of physiological shear force are likely to be much more relevant to the in vivo environment than those using anticoagulated blood and chemical platelet agonists [8-9]. We have demonstrated that shear-induced platelet reactivity tests in vitro, using non-anticoagulated blood, significantly correlate with the in vivo helium-neon (He-Ne) laserinduced thrombosis model in animals [10].

We have established in our laboratory a shear-induced platelet-rich thrombosis/coagulation test (Haemostatometry), a novel, commercially available shear-induced platelet-rich thrombosis/thrombolysis method (The Global Thrombosis Test, GTT) and a He-Ne laser-induced thrombosis model to investigate specific fruits and vegetables for antithrombotic activity. We have shown that different varieties of tomato, onion and strawberry can be classified according to shear-induced platelet-rich thrombotic activity (Haemostatometry), and that varieties of mulberry and carrot can be classified on this basis 
together with spontaneous thrombolytic activity (GTT) [11-15]. We have also demonstrated using Haemostatometry, that one onion variety cultivated in the northern part of Japan (Hokkaido) was notably antithrombotic [12] but the availability of this product remains limited. In the present study we have expanded our studies on the antithrombotic effects of onions using potentially more readily available onion varieties produced in the central area of Japan (Awaji).

\section{MATERIALS AND METHODS}

\subsection{Animals}

Male Wistar ST rats, at least 13 weeks old, and male C57BL/6 mice, 10 weeks old were purchased one week before use (Japan SLC Co. Ltd., Hamamatsu, Japan). Rats were fed a standard solid chow (MF, Oriental Yeast Co. Ltd., Osaka, Japan) and mice were similarly fed with standard solid chow (MF, Japan Clea CO. Ltd., Tokyo, Japan). Animals were allowed tap water ad libitum, and were maintained in compliance with the "Guiding Principles for the Care and Use of Animals in the field of Physiological Sciences," published by Physiological Society of Japan. The protocol was approved by the Animal Experiment Committee of Kobe Gakuin University. Animals were sacrificed using Somnopentyl following the experimental procedures.

\subsection{Onions}

Five varieties of onion, designated ONA-01, ONA-02, ONA-03, ONA-04 and ONA-05, were sown and harvested in August on the same test field of the Hyogo Prefectural Technology Center for Agriculture, Forestry and Fisheries, Awaji Agricultural Technology Institute, Awaji, Japan.

\subsection{Preparation of Onion Filtrate}

Six brown skin peeled bulbs per each variety were graded to a standard size using a plastic grader at room temperature to avoid inter-individual variation. The juice was centrifuged $\left(3000 \mathrm{rpm}, 15 \mathrm{~min}, 4^{\circ} \mathrm{C}\right)$ and the supernatant was filtered (FP30/5.0 CN-S, 5.0 $\mu \mathrm{m}$, Whatman PLC, Kent, UK). Clear filtrates were stored at $-80^{\circ} \mathrm{C}$ before use.

\subsection{In Vitro Assessment of Shear-Induced Platelet Reactivity and Spontaneous Thrombolytic Activity using the Global Thrombosis Test (GTT)}

The technique has been described in detail elsewhere [15-16]. The instrument was purchased from Montrose
Diagnostics Ltd., London, UK (www.globalthrombosis.com). Figures 1 and 2 illustrate the principles of the technique. A flat segment created along the inner wall of a conical

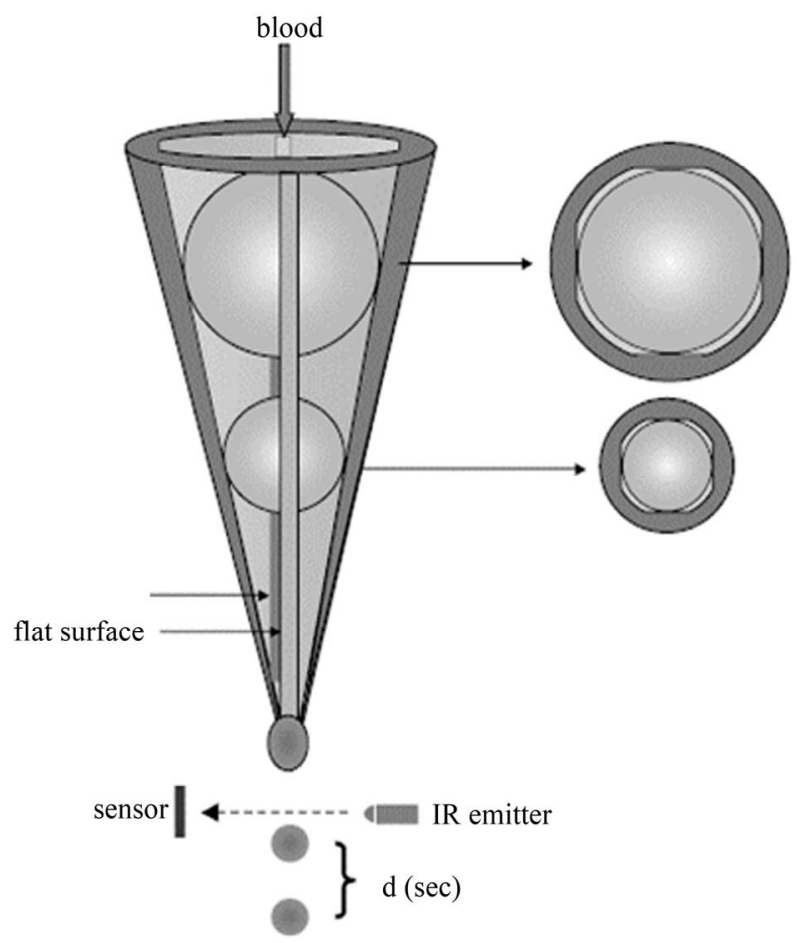

Figure 1. Schematic illustration of the GTT apparatus.

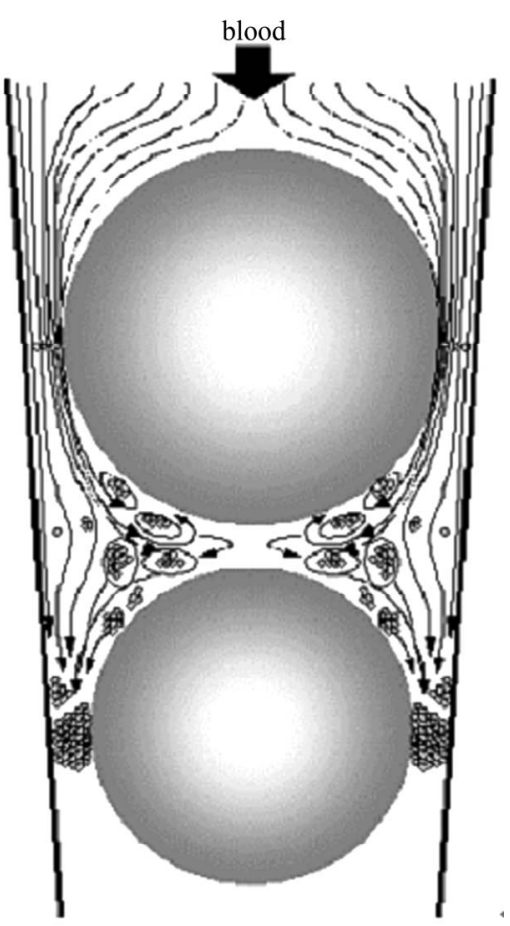

Figure 2. Schematic diagram showing the principle of the GTT. 
plastic tube forms the basis of the technique. When perfectly round steel ball-bearings are placed into the conical tube, the flat segment prevents the spheres from occluding the lumen. Blood is added, flows through the narrow gaps and exits in droplets into an adjacent collecting tube. The latter is trans-illuminated by a light emitter, and a sensor opposite the light source generates a signal whenever a drop of blood interrupts the light path. In essence, the instrument detects the time interval (d, sec) between consecutive blood drops. At the start, blood flow is rapid and hence (d) is small. Subsequently, the flow rate gradually decreases and hence $(d)$ increases. When the actual (d) exceeds 15 seconds (occlusion-d), the instrument displays "Occlusion Time (OT)", which is the time elapsed from the detection of the first drop of blood until (occlusion-d). Later, flow is completely arrested. Eventually, due to thrombolysis, flow is restored as indicated by the further detection of blood droplets. There is also an arbitrarily pre-set (d) (200 seconds) for lysis measurement (lysis-d). When (d) between the last drop before and the first drop after occlusion exceeds this (lysis-d), the instrument displays "Lysis Time (LT)". Hence, lysis time is calculated as follows: LT $=$ (time of first drop with $\mathrm{d}>$ (lysis-d) $)$ - (time of last drop with $\mathrm{d}<$ (lysis-d)). Blood flows by gravity at $37^{\circ} \mathrm{C}$ through the narrow gaps formed between the larger ball bearing and the inner wall of the tube, and the resulting high shear stress $\left(175 \mathrm{dyne} / \mathrm{cm}^{2}\right)$ activates platelets. These activated platelets remain single, since the very short transit time and high shear prevent aggregation. In contrast, in the space distal to the large ball bearing, i.e. between the two ball bearings, the low shear and turbulent flow favor large platelet aggregate formation. Furthermore, in this space between the ball bearings, activated platelets generate thrombin and initiate blood coagulation. Flow then carries these fibrin-stabilised platelet aggregates into the lower gaps where they are captured, resulting in occlusion and arrest of flow. Increased or decreased OT indicates inhibition or enhancement of platelet reactivity, respectively. Increased or decreased LT indicates inhibition or enhancement of spontaneous thrombolysis, respectively. Measurements were made six times $(n=6)$ in each sample.

Animals were fasted overnight but allowed water ad libitum. Blood was obtained from the abdominal aorta 30 minutes after anesthesia with sodium pentobarbital

(Somnopentyl, $64.8 \mathrm{mg} / \mathrm{ml}$, Kyoetsu Seiyaku Co. Ltd., Tokyo, Japan) diluted 5 times with saline $(65 \mathrm{mg} / \mathrm{kg}$, intramuscularly). Non-anticoagulated blood was mixed with saline (1:1). $3.6 \mathrm{ml}$ of the diluted blood and $0.4 \mathrm{ml}$ of onion filtrate or saline (control) (blood: filtrate $=9: 1$ ) were mixed in a syringe by inversion and the mixture was applied to GTT tube.

\subsection{In Vivo Assessment of the Antithrombotic Effects Using the Laser-Induced Thrombosis Test in Mice Carotid Arteries}

The He-Ne laser-induced platelet-rich thrombosis method has been previously described in detail $[17,18]$. Mice were anaesthetised with Somnopentyl $(65 \mathrm{mg} / \mathrm{kg}$, intramuscularly). A polyethylene tube (PE10, Becton Dickinson and Company, New Jersey, USA) was placed into the left femoral artery to inject dye and the carotid artery (450 - $500 \mu \mathrm{m}$ in diameter) was exposed by incision. The mouse was placed on a specially adapted microscope stage (Olympus Model BH-2, Olympus Co. Ltd., Tokyo, Japan) and Evans blue dye $(30 \mathrm{mg} / \mathrm{kg}$, Merck, Darnstadt, Germany) was injected intra-arterially. The centre of the exposed carotid artery was irradiated with a laser beam (Model Neo-50MS, $25 \mathrm{~mW}$ under an objective lens, Neoark Co. Ltd., Osaka, Japan). Thrombus formation at the site of irradiation was monitored under epi-illumination and simultaneously recorded on videotape using a CCD camera (Model TMC-7, Takenaka System Co. Ltd., Kyoto, Japan).

\subsection{Oral Administration of Onion Filtrates to Mice}

The vegetable filtrate or saline only (control) was administered through a gastric tube at $15.4 \mathrm{ml} / \mathrm{kg}$ (unheated filtrate) and 15.4 or $30.8 \mathrm{ml} / \mathrm{kg}$ (heated filtrate). Half volume of filtrate or saline $(7.7 \mathrm{ml} / \mathrm{kg}$ (unheated filtrate) and 7.7 or $15.4 \mathrm{ml} / \mathrm{kg}$ (heated filtrate) was repeatedly given at 30 minute interval as previously described [11]. The mouse was then anaesthetised and the thrombosis experiments commenced 90 min after the second oral administration. Antithrombotic or prothrombotic effects were assessed by estimating total thrombus size.

\subsection{Calculation of Thrombus Size}

Details of this technique have been described elsewhere [18]. Images of thrombus formation were computer-analysed at intervals of ten seconds. The area of thrombus was delineated and the mass of thrombus calculated by multiplying gray scale and area using Image $\mathrm{J}$ software (Image Processing and Analysis Java version 1.30, National Institutes of Health, Maryland, USA). Thrombotic status was expressed as the total sum of thrombus mass after the first 10 minutes of irradiation.

\subsection{Statistical Analysis}

Data from GTT were analysed by repeated ANOVA (General Linear Model), followed by the multiple comparison test (Dunnett), data from the laser-induced 
thrombosis experiments by the unpaired t-test. Values were expressed as means \pm SEM. $\mathrm{P}<0.05$ was considered as the limit of significance. Analyses were performed using Statistical Package Unistat 5.6 Light (London, UK).

\section{RESULTS}

\subsection{Effects of Raw Filtrates from Onion Varieties on Shear-Induced Platelet Thrombosis and Spontaneous Thrombolysis in Vitro}

The results are shown in Table 1. The filtrate from ONA-01 did not affect occlusion time (OT) but significantly inhibited spontaneous thrombolytic activity (LT), indicating that this variety had prothrombotic activity. ONA-02, ONA-04 and ONA-05 did not affect signifi- cantly either OT or LT. In contrast, the undiluted filtrate from ONA-03 significantly prolonged OT but did not affect LT, indicating that this particular variety had antithrombotic activity both in vitro and in vivo.

\subsection{Heat Stability of Antithrombotic Effect of ONA-03}

The antithrombotic effects of ONA-03 in vivo, were re-examined after the raw filtrate was heated in boiling water for 10 minutes. The filtrate was cooled to room temperature and the OT and LT were measured. The results are shown in Table 2. Similar results to those using unheated filtrate were obtained. The heated and undiluted (x1) filtrate significantly prolonged OT but had no significant effect on LT, again suggesting antithrombotic activity in vivo.

Table 1. Effects of raw filtrates from five onion varieties on shear-induced platelet thrombosis (occlusion time, OT) and spontaneous thrombolysis (lysis time, LT).

\begin{tabular}{|c|c|c|c|c|c|c|c|}
\hline $\begin{array}{l}\text { Variety } \\
\text { ONA-01 }\end{array}$ & $\begin{array}{l}\text { Dilution } \\
\text { control }\end{array}$ & 307.9 & \pm & 9.9 & 989.7 & \pm & 70.9 \\
\hline & $\times 3$ & 327.7 & \pm & 12.1 & 983.3 & \pm & 103.4 \\
\hline & $\times 10$ & 311.5 & \pm & 20.7 & 1024.3 & \pm & 46.5 \\
\hline \multirow{2}{*}{ ONA-02 } & $\times 3$ & 289.7 & \pm & 14.2 & 972.4 & \pm & 76.9 \\
\hline & $\times 10$ & 289.1 & \pm & 16.9 & 841.7 & \pm & 57.9 \\
\hline \multirow[t]{2}{*}{ ONA-03 } & control & 329.1 & \pm & 12.9 & 944.8 & \pm & 24.8 \\
\hline & $\times 1$ & 457.4 & \pm & $36.1^{* *}$ & 977.3 & \pm & 102.7 \\
\hline \multirow{3}{*}{ ONA-04 } & $\times 1$ & 272.9 & \pm & 25.1 & 1042.8 & \pm & 80.7 \\
\hline & $\times 3$ & 294.0 & \pm & 25.5 & 895.5 & \pm & 52.2 \\
\hline & $\times 10$ & 299.5 & \pm & 18.1 & 913.3 & \pm & 78.5 \\
\hline \multirow[t]{4}{*}{ ONA-05 } & control & 304.0 & \pm & 18.2 & 976.0 & \pm & 32.4 \\
\hline & $\times 1$ & 367.1 & \pm & 37.6 & 1032.4 & \pm & 102.3 \\
\hline & $\times 3$ & 341.9 & \pm & 19.4 & 891.8 & \pm & 26.6 \\
\hline & $\times 10$ & 311.7 & \pm & 14.9 & 889.3 & \pm & 56.7 \\
\hline
\end{tabular}

$* *: \mathrm{P}<0.01$

Table 2. Effects of heat treatment on the inhibitory activity of ONA-03 in shear-induced platelet thrombosis.

\begin{tabular}{cccccccc}
\hline Variety & Dilution factor of filtrate & \multicolumn{3}{c}{ Occlusion time } & \multicolumn{3}{c}{ Lysis time } \\
\hline ONA-03 & saline (control) & 302.7 & \pm & 12.5 & 1066.7 & \pm & 66.7 \\
& $\times 1$ & 446.0 & \pm & $19.4^{* *}$ & 1249.6 & \pm & 41.3 \\
& $\times 3$ & 345.6 & \pm & 9.3 & 981.5 & \pm & 75.0 \\
& $\times 10$ & 331.5 & \pm & 12.7 & 959.2 & \pm & 69.7 \\
\hline
\end{tabular}

\footnotetext{
$* *: \mathrm{P}<0.01$
} 


\subsection{Antithrombotic Activity of Orally Administered ONA-03}

Unheated or heated onion filtrate was orally administered to mice and the antithrombotic effect was assessed using the He-Ne laser-induced thrombosis test on exposed carotid arteries. Results are shown in Figure 3. Unheated and undiluted (x1) filtrate from ONA-03 significantly inhibited He-Ne laser-induced thrombosis (A). The same volume of heated filtrate did not inhibit thrombosis (B) but administration of twice the volume significantly inhibited thrombus formation (C). This inconsistency between the in vitro GTT test and the in vivo laser-induced thrombosis test might have been be due to different sensitivity of the methods or the effects of intestinal absorption. Nevertheless, the results demonstrated that the filtrate from ONA-03 was relatively heat stable.

\section{DISCUSSION}

Arterial platelet-rich thrombotic diseases are a social problem in many countries, and reliable tests to assess thrombotic tendency could be critically important in attempts to prevent these diseases. Various methods have been developed, including agonist-induced platelet aggregation tests using anticoagulated whole blood or platelet rich plasma. Biomarkers of coagulation and fibrinolysis have also been devised [19-24]. It is widely appreciated, however, that thrombus formation in vivo is governed by interactions between blood flow, blood components and the blood vessel wall as proposed by Virchow, and the pathophysiological relevance of these tests to thrombotic status in vivo is not clear. Alterna- tively, innovative tests, in which non-anticoagulated blood is used and platelets are activated by shear force under flow, have been proposed $[8,9,15]$. Clinical and experimental studies have suggested that these tests may be more relevant to thrombotic status in vivo $[8-10,16]$.

We have utilized in vivo tests of this nature to characterize various fruits and vegetables on the basis of antithrombotic activity. In particular, Haemostatometry, the GTT and the He-Ne laser-induced thrombosis test [17, 18] have enabled classification into different subgroups [11-14].

In an earlier study we used Haemostatometry and the He-Ne laser-induced thrombosis model to identify a specific antithrombotic onion variety produced in the northern part of Japan [12]. Distribution of this variety was limited, however, and further studies were devised to examine onion varieties from central Japan (Awaji Island). These varieties were potentially more available than others, and were classified using the GTT in place of Haemostatometry.

The principle aim of our current series of investigations is to broadly identify fruits and vegetables with antithrombotic activity. In addition, thrombus evolution is largely determined by a balance between pro-aggregatory and procoagulant mechanisms on the one hand, and fibrinolytic processes on the other [25], and the relationship between the concentration of specific antithrombotic substances and overall antithrombotic activity of fruits and vegetables remains to be defined. In this respect, quercetin is reported to be important in inhibiting collagen-stimulated platelet aggregation [26-28], but a high concentration of quercetin in onions does not necessarily mean that onions are antithrombotic, and we

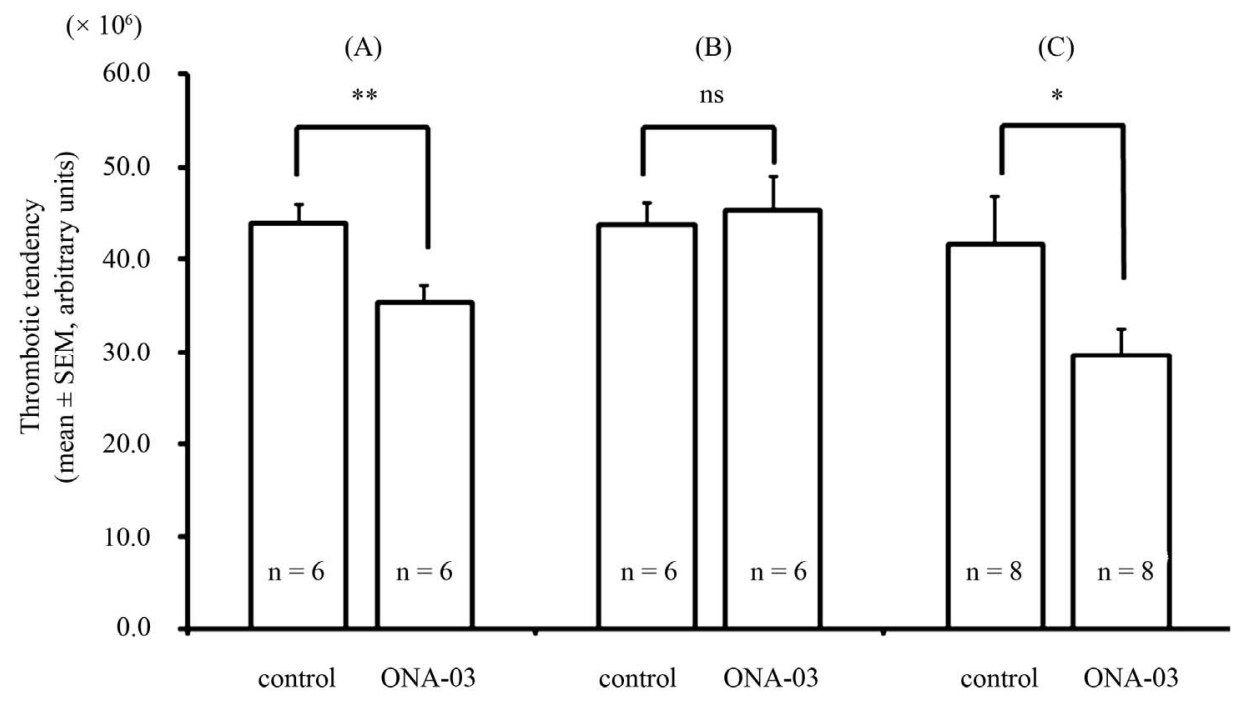

Figure 3. Antithrombotic activity of unheated and heated onion filtrates from ONA-03 n: number of measurements; *: $\mathrm{P}<0.05, * *$ : $\mathrm{P}<0.01$. 
have previously demonstrated that there was no significant correlation between quercetin concentration of onions and overall antiplatelet activity using shear-induced platelet function tests in vitro (Haemostatometry; 12). Furthermore, the fibrinolytic properties of onions have been the focus of some antithrombotic studies [29] but significant fibrinolytic activity was not evident in any of the five varieties of onions used in the present investigations. Moreover, for clinical purposes it is important to note that the amounts of onion extracts given to animals in the present studies would be approximately equivalent to $40 \mathrm{~kg}$ onion given to a person with a body weight $70 \mathrm{~kg}$. This is clearly impracticable, and further studies are required to identify and isolate antithrombotic substances that could be scientifically important and lead to significant clinical benefit. Detailed analysis of this nature is outside the scope of the present studies, but nevertheless, our characterization of particular prothrombotic and antithrombotic fruits and vegetables could lead to the development of novel therapeutic products to help to prevent arterial thrombotic diseases.

\section{REFERENCES}

[1] Lichtenstein, A.H., Appel, L.J., Brands, M., Carnethon, M., Daniels, S., Franch, H.A., Franklin, B., Kris-Etherton, P., Harris, W.S., Howard, B., Karanja, N., Lefevre, M., Rudel, L., Sacks, F., Van Horn, L., Winston, M. and Wylie-Rosett, J. (2006) Summary of american heart association diet and lifestyle recommendations revision 2006. Arteriosclerosis Thrombosis and Vascular Biology, 26, 2186-2191. doi:10.1161/01.ATV.0000238352.25222.5e

[2] Ulbricht, T.L. and Southgate, D.A. (1991) Coronary heart disease: seven dietary factors. Lancet, 338, 985- 992. doi:10.1016/0140-6736(91)91846-M

[3] Renaud, S. and de Lorgeril, M. (1992) Wine, alcohol, platelets, and the French paradox for coronary heart disease. Lancet, 339, 1523-1526. doi:10.1016/0140-6736(92)91277-F

[4] Joshipura, K.J., Ascherio, A., Manson, J.E., Stampfer, M.J., Rimm, E.B., Speizer, F.E., Hennekens, C.H., Spiegelman, D. and Willett, W.C. (1999) Fruit and vegetable intake in relation to risk of ischemic stroke. Journal of the American Medical Association, 282, 1233-1239. doi:10.1001/jama.282.13.1233

[5] Liu, S., Manson, J.E., Lee, I.M., Cole, S.R., Hennekens, C.H., Willett, W.C. and Buring, J.E. (2000) Fruit and vegetable intake and risk of cardiovascular disease: the women's health study. American Journal of Clinical Nutrition, 72, 922-928.

[6] Joshipura, K.J., Hu, F.B., Manson, J.E., Stampfer, M.J., Rimm, E.B., Speizer, F.E., Colditz, G., Ascherio, A., Rosner, B., Spiegelman, D. and Willett W.C. (2001) The effect of fruit and vegetable intake on risk for coronary heart disease. Annals of Internal Medicine, 134, 1106 -1114 .
[7] Bazzano L.A, He J, Ogden L.G, Loria C.M, Vupputuri S, Myers L. and Whelton P.K (2002) Fruit and vegetable intake and risk of cardiovascular disease in US adults: the first national health and nutrition examination survey epidemiologic follow-up study. American Journal of Clinical Nutrition, 76, 93-99.

[8] Ratnatunga, C.P., Edomondson, S.F., Rees, G.M. and Kovacs, I.B. (1992) High-dose aspirin inhibits shear-induced platelet reaction involving thrombin generation. Circulation, 85, 1077-1082.

[9] Gorog, D.A. and Kovacs, I.B. (1995) Thrombotic status analyser. Journal of Thrombosis and Haemostasis, 73, 514-520.

[10] Yamamoto, J. (2007) Prediction of thrombotic status by a new test and prevention of thrombotic disorders by foods with antithrombotic activity. The $7^{\text {th }}$ TTM Forum Report 2007, 203-220, (Japanese).

[11] Yamamoto, J., Taka, T., Yamada, K., Ijiri, Y., Murakami, M., Hirata, Y., Naemura, A., Hashimoto, M., Yamashita, T., Oiwa, K., Seki, J., Suganuma, H., Inakuma, T. and Yoshida, T. (2003) Tomatoes have natural antithrombotic effects. British Journal of Nutrition, 90, 1031-1038. doi:10.1079/BJN2003994

[12] Yamada, K., Naemura, A., Sawashita, N., Noguchi, Y. and Yamamoto, J. (2004) An onion variety has natural antithrombotic effect as assessed by thrombosis/thrombolysis. Thrombosis Research, 114, 213-220. doi:10.1016/j.thromres.2004.06.007

[13] Naemura, A., Mitani, T., Ijiri, Y., Tamura, Y., Yamashita, T., Okimura, M. and Yamamoto, J. (2005) Anti-thrombotic effect of strawberries. Blood Coagulation \& Fibrinolysis, 16, 501-509. doi:10.1097/01.mbc.0000184737.50594.a8

[14] Yamamoto, J., Naemura, A., Ura, M., Ijiri, Y., Yamashita, T., Kurioka, A. and Koyama, A. (2006) Testing various fruits for anti-thrombotic effect: I. Mulberries. Platelets, 17, 555-564. doi:10.1080/09537100600759295

[15] Yamamoto, J., Yamashita, T., Ikarugi, H., Taka, T., Hashimoto, M., Ishii, H., Watanabe, S. and Kovacs, I.B. (2003) Gorog Thrombosis Test: a global in-vitro test of platelet function and thrombolysis. Blood Coagulation \& Fibrinolysis, 14, 31-39. doi:10.1097/00001721-200301000-00007

[16] Saraf, S., Wellsted, D., Sharma, S. and Gorog, D.A. (2009) Shear-induced global thrombosis test of native blood: pivotal role of ADP allows monitoring of P2Y12 antagonist therapy. Thrombosis Research, 124, 447- 451. doi:10.1016/j.thromres.2009.04.013

[17] Kovacs, I.B., Tigyi-Sebes, A., Trombitas, K. and Gorog, P. (1975) Evans blue: an ideal energy-absorbing material to produce intravascular microinjury by $\mathrm{He}-\mathrm{Ne}$ gas laser. Microvascular Research, 10, 107-124. doi:10.1016/0026-2862(75)90025-4

[18] Ijiri, Y., Miura, M., Hashimoto, M., Fukunaga, C., Watanabe, S., Kubota, A., Oiwa, K., Okuda, T. and Yamamoto, J. (2002) A new model to evaluate the diet-induced prothrombotic status, using He-Ne laser-induced thrombogenesis in the carotid artery of apolipoprotein E-deficient and low-density lipoprotein receptor-deficient mice. Blood Coagulation \& Fibrinolysis, 13, 497-504. doi:10.1097/00001721-200209000-00004 
[19] Nakajima, S., Noguchi, T., Taka, T., Ueda, T., Kaizu, K., Fukamizu, M., Fujita, S., Tabuchi, M. and Yamamoto, J. (2000) A global platelet test of thrombosis and thrombolysis detects a prothrombotic state in some patients with non-insulin dependent diabetes and in some patients with stroke. Platelets, 11, 459-466. doi:10.1080/09537100020027833

[20] Harrison, P. (2005) Platelet function analysis. Blood Reviews, 19, 111-123. doi:10.1016/j.blre.2004.05.002

[21] Ferreiro, J.L., Sibbing, D. and Angiolillo, D.J. (2010) Platelet function testing and risk of bleeding complications. Thrombosis and Haemostasis, 103, 1128-1135. doi:10.1160/TH09-11-0799

[22] Gorog, D.A. (2010) Prognostic value of plasma fibrinolysis activation markers in cardiovascular disease. Journal of the American College of Cardiology, 55, 2701-2709. doi:10.1016/j.jacc.2009.11.095

[23] Lippi, G., Cervellin, G., Franchini, M. and Favaloro, E.J. (2010) Biochemical markers for the diagnosis of venous thromboembolism: the past, present and future. Journal of Thrombosis and Thrombolysis, 30, 459-471. doi:10.1007/s11239-010-0460-x

[24] Saraf, S., Christopoulos, C., Salha, I.B., Stott, D.J. and Gorog, D.A. (2010) Impaired endogenous thrombolysis in acute coronary syndrome patients predicts cardiovascular death and nonfatal myocardial infarction. Journal of the American College of Cardiology, 55, 2107- 2115. doi:10.1016/j.jacc.2010.01.033
[25] Bodary, P.F., Wickenheiser, K.J. and Eitzman, D.T. (2002) Recent advances in understanding endogenous fibrinolysis: implications for molecular-based treatment of vascular disorders. Expert Reviews in Molecular Medicine, 4, $1-10$ doi:10.1017/S1462399402004362

[26] Hubbard, G.P., Stevens, J.M., Cicmil, M., Sage, T., Jordan, P.A., Williams, C.M., Lovegrove, J.A. and Gibbins, J.M. (2003) Quercetin inhibits collagen-stimulated platelet activation through inhibition of multiple components of the glycoprotein VI signaling pathway. Journal of Thrombosis and Haemostasis, 1, 1079-1088. doi:10.1046/j.1538-7836.2003.00212.x

[27] Hubbard, G.P., Wolffram, S., Lovegrove, J.A. and Gibbins, J.M. (2004) Ingestion of quercetin inhibits platelet aggregation and essential components of the collagenstimulated platelet activation pathway in humans. Journal of Thrombosis and Haemostasis, 2, 2138-2145. doi:10.1111/j.1538-7836.2004.01067.x

[28] Hubbard, G.P., Wolffram, S., de Vos, R., Bovy, A., Gibbins, J.M. and Lovegrove, J.A. (2006) Ingestion of onion soup high in quercetin inhibits platelet aggregation and essential components of the collagen-stimulated platelet activation pathway in man: a pilot study. British Journal of Nutrition, 96, 482-488.

[29] Nagda, K.K., Ganeriwal, S.K., Nagda, K.C. and Diwan, A.M. (1983) Effect of onion and garlic on blood coagulation and fibrinolysis in vitro. Indian Journal of Physiology \& Pharmacology, 27, 141-145. 